\title{
Social media and the intrauterine device: a YouTube content analysis
}

\author{
Brian T Nguyen, ${ }^{1,2}$ Allison J Allen ${ }^{2}$
}

\begin{abstract}
'Department of Obstetrics and Gynecology, University of Southern California, Los Angeles, California, USA

${ }^{2}$ Department of Obstetrics and Gynecology, Oregon Health \& Science University, Portland, Oregon, USA
\end{abstract}

\section{Correspondence to} Dr Brian T Nguyen, Department of Obstetrics and Gynecology, University of Southern California, Los Angeles, CA 90033, USA; nguyenbt@usc.edu

Received 15 April 2017 Revised 3 October 2017 Accepted 25 October 2017 Published Online First 23 November 2017

\section{CrossMark}

To cite: Nguyen BT, Allen AJ. BMJ Sex Reprod Health 2018;44:27-31.

\begin{abstract}
Background YouTube's online archive of video testimonials related to health information are more commonly viewed than those developed by clinicians and professional groups, suggesting the importance of the patient experience to viewers. We specifically sought to examine the accuracy of information on, and projected acceptability of, the intrauterine device (IUD) from these YouTube testimonials.

Methods We searched YouTube for videos about individual uploaders' IUD experiences, using the search terms 'intrauterine device', 'IUD', 'Mirena' and 'Paragard'. Given interest in

Key messages

- YouTube testimonials provide a readily accessible source of information and vicarious experience to women who may be considering an IUD for contraception.

- In this analysis, one-third of IUD user testimonials contained inaccurate information and two-thirds mentioned side effects.

- Nevertheless, the majority of IUD experiences uploaded to YouTube were perceived by our study viewers to be positive.
\end{abstract} user testimonials, we excluded professional and instructional videos belonging to commercial or non-profit entities. Two reviewers independently analysed the videos using a structured guide, with attention to inaccurate information.

Results Of 86 identified videos, four videos featured clinicians and were excluded; 62 met inclusion criteria. Interrater agreement on IUD portrayal was good $(K=0.73)$. Young (mean age 25 , range $19-38$, years), white $(75 \%)$, nulliparous (61\%) women primarily uploaded content. Most described placement of the LNG-IUS (65\%), were posted within 1 month of insertion (45\%), and mentioned side effects $(66 \%)$ - bleeding, pain, and partner sensation of the strings. About one-third of videos contained inaccurate information (34\%) and were thought to project an overall negative experience (30\%). Videos portraying IUDs negatively were associated with inaccurate information and/or mention of side effects.

Conclusion While one-third of IUD user testimonials on YouTube contained inaccurate information, the majority of IUD experiences were perceived by our study viewers to be positive.

\section{INTRODUCTION}

Intrauterine devices (IUDs) offer safe, effective, long-term contraception and should be considered for all women who seek reliable, reversible contraception, ${ }^{1}$ including adolescent women. ${ }^{2}$ However, IUDs are relatively underutilised, representing only 5.5\% of contracepting women in the USA ${ }^{3}$ and $1.0 \%$ of contracepting adolescents ${ }^{4}$ from 2006 to 2008. Lack of awareness or knowledge of the IUD and high upfront costs have been cited as barriers to IUD uptake. ${ }^{5}$ However, even after removing all barriers, as in the Contraceptive CHOICE project, where women seeking year-long contraception were provided with comprehensive information and individual counselling on IUDs, as well as cost-free contraception, $55 \%$ of participants still declined placement. ${ }^{6}$ This finding may be related to sociocultural biases underlying the IUD's acceptability; biases may be perpetuated by anecdotal experiences or misleading testimonials from young women's social networks, which can be powerfully influential. ${ }^{7}$ Testimonials may be perceived as more relevant than the impartial recommendations of healthcare providers, who patients may perceive as lacking the same perspective and degree of personal investment. ${ }^{89}$

IUD experiences among one's social network, however, may be a limited resource that is not always available to women of reproductive age. The internet remains one of the most accessible and affordable sources of information for women. The Pew Internet Project revealed that nearly three-quarters of 
Americans search the internet for health information, including contraceptive information, with more than half reporting a positive impact of the internet on their healthcare decisions. ${ }^{10}$ The internet is the primary source of contraceptive information among more than $50 \%$ of young adults. ${ }^{10}$ YouTube (https:// www.YouTube.com), an online platform for sharing videos, is one of the internet's most frequently visited websites. YouTube's diverse content includes women's IUD experiences. Of note, the majority of YouTube's videos are uploaded by individuals, and previous examinations of health-related topics on YouTube note that they generally receive more views than those uploaded by professional societies. ${ }^{11}$ Consequently, the opinions and user experiences of video uploaders may be more valuable than verified content, and play a role in contraceptive decision-making. While YouTube is not the only freely accessible social media outlet (others include Instagram, Snapchat, Twitter and Facebook), YouTube is unique among these outlets given that all videos are public and searchable both on YouTube and via the Google search engine. Instagram, Snapchat and Facebook require varying degrees of uploader permissions before videos can be viewed; furthermore, videos on Snapchat are deleted on viewing by the intended recipient. No studies have specifically described independent, IUD-related content on YouTube or determined the extent to which they perpetuate inaccurate information. We performed a content analysis of online testimonials specific to the IUD.

\section{METHODS}

We conducted a search for IUD testimonials on YouTube during the first week of January 2012, using the search terms: 'intrauterine device' and 'IUD'. We included the terms 'Mirena' and 'Paragard' given anecdotal experience of patients being unfamiliar with the term 'IUD' and their preference to use brand names for contraceptive methods. We excluded videos if they were: (1) not in English; (2) duplicate entries; (3) uploaded by a business or group; (4) produced by professional production companies for educational/ promotional use; (5) did not relate to the actual experience of having an IUD in place, (eg, requests for information, pre-insertion contemplation); or (6) of audio/video quality that did not allow our reviewers to understand and analyse their content. We limited our search results to the first 60 videos (three pages), as $90 \%$ of internet users reportedly rely on results from the first three pages of a web search. ${ }^{12}$ Given concern that videos might be taken down during the time in which we were performing this research, all videos were downloaded during the week of our search. As YouTube search results do not group videos by uploader, we independently evaluated videos that depicted IUD experiences from the same uploader at different time points. Oregon Health \& Science
University's Institutional Review Board declared this study design as non-human subjects research.

The research staff developed and used a standard instrument to record information about each YouTube video. The staff examined each video for uploader demographics provided on the uploader's public profile page, number of video views, IUD type, subjective insertion experience, mention of pain/side effects, and presence/absence of inaccurate information about the IUD. The staff also examined each video's potential influence or reach, which was defined as lower or higher than the median number of view counts over length of time online (days). The primary clinician (BN) subsequently checked uploader claims about the IUD, using the WHO's Selected Practice Recommendations for Contraceptive Use $(2005)^{13}$ and Committee opinions from the American College of Obstetricians and Gynecologists ${ }^{14}$ as references.

Given the subjectivity entailed in the assessment of each video testimonial's portrayal of the IUD, two independent viewers from both clinical (AA) and non-clinical ( $\mathrm{RN})$ backgrounds analysed the videos. A third viewer (BN) arbitrated any differences. The staff categorised videos as negative if the perceived central message suggested that IUD use was not satisfactory or acceptable (eg, emphasis on severe pain, cramping, loss/expulsion, migration, heavy bleeding, etc.). They categorised videos as positive if the central message supported IUD use (eg, less bleeding, long-acting, reversibility, discretion, etc). Videos discussing negative experiences or side effects that ultimately recommended the IUD's use were categorised as positive.

We calculated descriptive statistics and performed bivariate analyses. We determined interrater agreement on IUD portrayal using Kappa coefficients. We subsequently compared uploader demographics and video characteristics against overall IUD portrayal and view counts/day online, using Chi-square tests with significance at the $\mathrm{P}<0.05$ level. We recorded and analysed all data using SPSS (SPSS Statistics for Windows, Version 21.0, NY).

\section{RESULTS}

Of 86 identified videos, four videos featured clinicians and were excluded; 62 (72\%) met inclusion criteria (table 1). The earliest video of a user's experience with the IUD was uploaded in April of 2008. Mean video length was 6 min; median time online was 525 days; median view count was five times/day. The content was primarily uploaded by young (mean age 25 , range 19-38, years), white (74\%) women. Among videos mentioning when the uploader had her IUD inserted, nearly half $(45 \%, 23 / 51)$ of women posted their video within 1 month of insertion. Approximately one-third of videos contained inaccurate information. Examples of these claims included that the IUD could not be used by adolescents and nulliparous women, that IUD users experienced delayed return to fertility 
Table 1 Associations of YouTube video impact and intrauterine device (IUD) portrayal with uploader and video characteristics

\begin{tabular}{|c|c|c|c|c|c|c|c|}
\hline \multirow[b]{2}{*}{ Uploader and video characteristics } & \multirow{2}{*}{$\begin{array}{l}\text { Total } \\
\text { (n (\%)) }\end{array}$} & \multicolumn{2}{|c|}{$\begin{array}{l}\text { Video influence } \\
\text { (view counts/day) (n (\%)) }\end{array}$} & \multirow[b]{2}{*}{$\mathbf{P}$} & \multicolumn{2}{|c|}{$\begin{array}{l}\text { Perceived IUD portrayal } \\
\text { (n (\%)) }\end{array}$} & \multirow[b]{2}{*}{$\mathbf{P}$} \\
\hline & & $\leq 5$ & $>5$ & & Positive & Negative & \\
\hline \multicolumn{8}{|l|}{ Time online (days) } \\
\hline$>365$ & $48(77.4)$ & $20(41.7)$ & $28(58.3)$ & 0.07 & $35(72.9)$ & $13(27.1)$ & 0.91 \\
\hline$\leq 365$ & $14(22.6)$ & $10(71.4)$ & $4(28.6)$ & & $10(71.4)$ & $4(28.6)$ & \\
\hline \multicolumn{8}{|l|}{ Video length (min) } \\
\hline$>5$ & $33(53.2)$ & $17(51.5)$ & $16(48.5)$ & 0.60 & $22(66.7)$ & $11(33.3)$ & 0.27 \\
\hline$\leq 5$ & $29(46.8)$ & $13(44.8)$ & $16(55.2)$ & & $23(79.3)$ & $6(20.7)$ & \\
\hline \multicolumn{8}{|l|}{ Uploader age (years) } \\
\hline$\geq 25$ & $27(50.9)$ & $11(42.3)$ & $15(57.7)$ & 0.88 & $15(57.7)$ & $11(42.3)$ & 0.03 \\
\hline$<25$ & $26(49.1)$ & $12(44.4)$ & $15(55.6)$ & & $23(85.2)$ & $4(14.8)$ & \\
\hline \multicolumn{8}{|l|}{ Uploader race/ethnicity } \\
\hline White & $46(74.2)$ & $21(45.7)$ & $25(54.3)$ & 0.47 & $35(76.1)$ & $11(23.9)$ & 0.29 \\
\hline Other & $16(25.8)$ & $9(56.3)$ & $7(43.8)$ & & $10(62.5)$ & $6(37.5)$ & \\
\hline \multicolumn{8}{|l|}{ IUD type } \\
\hline Mirena & $39(65.0)$ & $14(35.9)$ & $25(64.1)$ & 0.38 & $26(66.7)$ & $13(33.3)$ & 0.71 \\
\hline Paragard & $21(35.0)$ & $10(47.6)$ & $11(52.4)$ & & $15(71.4)$ & $6(28.6)$ & \\
\hline \multicolumn{8}{|c|}{ Interval between IUD insertion and video upload (days) } \\
\hline$<1$ month & $23(45.1)$ & $14(60.9)$ & $9(39.1)$ & 0.20 & $19(82.6)$ & $4(17.4)$ & 0.03 \\
\hline 1 month-1 year & $19(37.3)$ & $13(68.4)$ & $6(31.6)$ & & $12(63.2)$ & $7(36.8)$ & \\
\hline$\geq 1$ year & $9(17.6)$ & $3(33.3)$ & $6(66.7)$ & & $3(33.3)$ & $6(66.7)$ & \\
\hline \multicolumn{8}{|l|}{ False claims } \\
\hline Yes & $21(33.9)$ & $10(47.6)$ & $11(52.4)$ & 0.93 & $11(52.4)$ & $10(47.6)$ & 0.01 \\
\hline No & $41(66.1)$ & $20(48.8)$ & $21(51.2)$ & & $34(82.9)$ & $7(17.1)$ & \\
\hline \multicolumn{8}{|l|}{ Side effects mentioned } \\
\hline Yes & $41(66.1)$ & $21(51.2)$ & $20(48.8)$ & 0.53 & $24(58.5)$ & $17(41.5)$ & 0.00 \\
\hline No & $21(33.9)$ & $9(42.9)$ & $12(57.1)$ & & $21(100.0)$ & $0(0.0)$ & \\
\hline \multicolumn{8}{|l|}{ IUD portrayal } \\
\hline Positive & $45(72.6)$ & $20(51.3)$ & $19(48.7)$ & 0.28 & - & - & - \\
\hline Negative & $17(27.4)$ & $7(35.0)$ & $13(65.0)$ & & - & - & \\
\hline
\end{tabular}

following removal, and could have severe systemic side effects from exposure to copper or progestogens. Side effects were described in two-thirds of videos; the most frequently mentioned included post-insertion pain $(24 \%, 15 / 62)$, irregular bleeding $(24 \%, 15 / 62)$, and partner sensation of the strings $(8 \%, 5 / 62)$. Removal was mentioned in five videos (8\%). Portrayals of the IUD were rated as generally positive $(68-73 \%$ ) by our raters. Interrater agreement on the negative/positive portrayal of the IUD for each video was good $(\mathrm{K}=0.73$, $\mathrm{P}<0.001,95 \%$ CI 0.54 to 0.92 ).

Table 1 provides bivariate associations of video characteristics with perceived IUD portrayal and potential viewer influence. Video and uploader characteristics were not associated with view count, except for receipt of a copper IUD, which was associated with more views overall. Negative portrayals of the IUD were associated with uploaders older than age 25 years, inaccurate claims, and mention of side effects. IUD portrayals were not associated with potential viewer influence.

\section{DISCUSSION}

Given the same information on safety and efficacy of the IUD, reproductive-age women are still less likely than their gynaecologist counterparts to use an IUD for contraception; ${ }^{15}$ this disparity may be related to the vicarious experience that women's healthcare providers acquire during their clinical encounters. To better reassure themselves or validate their concerns about the IUD, women who are less familiar with the IUD may rely on video testimonials that are readily available on YouTube and accessible via the Google search engine. These women represent a population at risk of using less effective contraceptive methods or discontinuation if not met by personal, reassuring and medically accurate video testimonials. 
In our study we found a substantial number of regularly viewed IUD testimonials on YouTube, the majority of which contained accurate information. About one-third of the videos made inaccurate claims, a finding not unlike other reviews of webpage-based medical information on the internet, ${ }^{16}$ even specific to the IUD. ${ }^{17}$ Specific side effects (bleeding, pain, partner sensation of strings) were also commonly mentioned and at similar proportions to those reported in actual clinical encounters. ${ }^{18}$ Yet while the inclusion of inaccurate claims and side effects was associated with the IUD's overall portrayal, the majority of IUD testimonials were still perceived as positive by our raters and neither characteristic was significantly linked to video view counts and potential influence. These findings are consistent with those of another study examining the content of YouTube videos on the single-rod contraceptive implant, which rated $61 \%$ of testimonials as positive and noted that only the minority contained misinformation. ${ }^{19}$ With respect to negative portrayals of the IUD, we noted that they were more commonly identified among videos created more than a year after a user had her IUD inserted. This finding suggests an uploader bias where users were only prompted to create a video so remote from their IUD insertion if they had recently experienced and identified a problem.

While professional videos were excluded from our analysis, we noted disproportionately fewer videos from clinicians and their professional societies. Encouraging healthcare providers to create videos that address erroneous claims, advocate for more effective contraceptive methods, and alleviate patient anxieties may be helpful in increasing uptake. ${ }^{20}$ However, the impact of clinician videos may be limited, as previous studies of healthcare information on YouTube noted that testimonials were more frequently viewed over lectures or public service announcements from professional societies, irrespective of credibility. ${ }^{11}$ Nevertheless, view counts may not accurately reflect a video's potential influence, as they do not account for incomplete views where the viewer may neither watch the entire video nor incorporate the information or perspective into healthcare decision-making. Additionally, positive perceptions of the IUD as rated by our reviewers may not mirror the actual perceptions of YouTube viewers. While we used predetermined evaluation criteria, obtained good interrater agreement, and employed student reviewers whose ages were expected to closely match the demographic of YouTube users, we could not guarantee that they both were unbiased about the IUD. These findings highlight both a limitation of our study and an opportunity without collecting data from viewers, we are unable to discuss the impact of misinformation or the IUD's portrayal on viewer knowledge, interest and uptake of the IUD. Consequently, recommendations for the use of social media as a tool to promote contraception may thus benefit from directly asking women seeking contraception about which social media outlets they used when looking for information, and the characteristics of those media that were helpful for decision-making purposes.

We acknowledge the limits of attempting to provide a complete examination of IUD testimonials on YouTube. Limitations of the YouTube search engine at the time of data collection prevented the inclusion of relevant videos with misspelled titles. Additionally, given that online content expands by the minute, the findings discussed are unlikely to be generalisable to content found online in the future. In fact, by the time we collected and analysed the IUD testimonials that were discussed, three levonorgestrel IUDs entered the market - Skyla, Kyleena and Liletta — such that the environment of IUD testimonials is likely to be different from that which was found during our original search. Nevertheless, the insights presented provide a baseline understanding and may inform future interventions or research that utilise this medium of information dissemination.

Acknowledgements The authors would like to acknowledge Dr Jeffrey T Jensen and Raji Nagalla for their contributions to the data collection and interpretation. The authors also thank Dr Melissa J Chen for her assistance with manuscript revisions.

Contributors All the authors contributed to the manuscript as follows: BTN: conceived the study, designed the protocol, developed analysis tools and databases, obtained IRB approval, collected all data, oversaw video interpretation, analysed data, participated in abstract writing, authored the manuscript, and completed all revision requests. BTN is responsible for the overall content as guarantor. AJA: assisted with setting up the database for data collection, interpreted videos, participated in analysis and interpretation of data, led abstract writing, and reviewed the manuscript in its early stages.Collaborators: JTJ: provided initial oversight of the study design and interpretation of the data, as well as providing insights relevant to manuscript writing. RN: helped to analyse video data.

Disclaimer The study findings described in this article have not been published in any other journal.

\section{Competing interests None declared.}

Ethics approval Oregon Health \& Science University.

Provenance and peer review Not commissioned; externally peer reviewed.

Data sharing statement All the videos examined in this study are available publicly on YouTube.com.

(C) Article author(s) (or their employer(s) unless otherwise stated in the text of the article) 2018. All rights reserved. No commercial use is permitted unless otherwise expressly granted.

\section{REFERENCES}

1 American College of Obstetricians and Gynecologists (ACOG) Committee on Practice Bulletins - Gynecology. ACOG Practice Bulletin. Clinical management guidelines for obstetriciangynecologists. Number 59, January 2005. Intrauterine device. Obstet Gynecol 2005;105:223-32.

2 Allen S, Barlow E. Long-acting reversible contraception: an essential guide for pediatric primary care providers. Pediatr Clin North Am 2017;64:359-69. 
3 Kavanaugh ML, Jerman J, Hubacher D, et al. Characteristics of women in the United States who use long-acting reversible contraceptive methods. Obstet Gynecol 2011;117:1349-57.

4 Ott MA, Sucato GS. Committee on Adolescence. Contraception for adolescents. Pediatrics 2014;134:e1257-81.

5 American College of Obstetricians and Gynecologists Committee on Gynecologic Practice, Long-Acting Reversible Contraception Working Group. ACOG Committee Opinion No. 450: Increasing use of contraceptive implants and intrauterine devices to reduce unintended pregnancy. Obstet Gynecol 2009;114:1434-8.

6 Secura GM, Allsworth JE, Madden T, et al. The contraceptive CHOICE Project: reducing barriers to long-acting reversible contraception. Am J Obstet Gynecol 2010;203:115.e1-7.

7 Yee L, Simon M. The role of the social network in contraceptive decision-making among young, African American and Latina women. J Adolesc Health 2010;47:374-80.

8 Fleming KL, Sokoloff A, Raine TR. Attitudes and beliefs about the intrauterine device among teenagers and young women. Contraception 2010;82:178-82.

9 Rubin SE, Winrob I. Urban female family medicine patients' perceptions about intrauterine contraception. $J$ Womens Health 2010;19:735-40.

10 Fox S, Jones S. The Social Life of Health Information: Americans' pursuit of health takes place within a widening network of both online and offline sources. Pew Research Center. Pew Internet \& American Life Project, 2010. Reports/2009/8-The-Social-Life-of-Health-Information.aspx.

11 Pant S, Deshmukh A, Murugiah K, et al. Assessing the credibility of the "YouTube approach" to health information on acute myocardial infarction. Clin Cardiol 2012;35:281-5.
12 Rieger OY. Search engine use behavior of students and faculty: User perceptions and implications for future research. First Monday 2009;14.

13 World Health Organization. Selected practice recommendations for contraceptive use. World Health Organization, 2005.

14 Committee on Gynecologic Practice Long-Acting Reversible Contraception Working Group. Committee Opinion No. 642: Increasing access to contraceptive implants and intrauterine devices to reduce unintended pregnancy. Obstet Gynecol 2015;126:e44-8.

15 Stern LF, Simons HR, Kohn JE, et al. Differences in contraceptive use between family planning providers and the U.S. population: results of a nationwide survey. Contraception 2015;91:464-9.

16 Culver JD, Gerr F, Frumkin H. Medical information on the Internet: a study of an electronic bulletin board. J Gen Intern Med 1997;12:466-70.

17 Weiss E, Moore K. An assessment of the quality of information available on the internet about the IUD and the potential impact on contraceptive choices. Contraception 2003;68:35964.

18 Lara-Torre E, Spotswood L, Correia N, et al. Intrauterine contraception in adolescents and young women: a descriptive study of use, side effects, and compliance. J Pediatr Adolesc Gynecol 2011;24:39-41.

19 Paul J, Boraas CM, Duvet M, et al. YouTube and the single-rod contraceptive implant: a content analysis. J Fam Plann Reprod Health Care 2017;43:195-200.

20 Topps D, Helmer J, Ellaway R. YouTube as a platform for publishing clinical skills training videos. Acad Med 2013;88:192-7.

\section{FSRH Webinars on Demand}

\begin{abstract}
Did you know that FSRH offers free webinars for members?
\end{abstract}

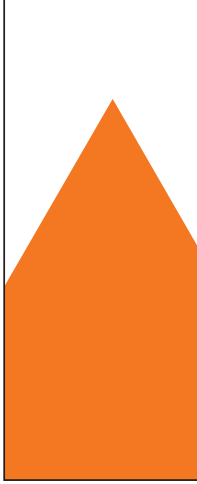

Topics include:

- Contraception for Women over 40 years

- Quick Starting Contraception

- Emergency Contraception

- Contraception Following Pregnancy

- An Update on the UKMEC 2016

- Drug Interactions for Hormonal Contraception

FSRH members can log into the website at www.fsrh.org/webinars to access these for free any time.

Non FSRH members can pay per view by visiting: http://events4healthcare.com/fsrh/webinars/ 